\title{
Asymptotic Rejection of a Class of Persistent Disturbances for Spacecraft Systems
}

\author{
Zhiyong Chen and Jie Huang
}

\begin{abstract}
In this paper, we present a global robust adaptive regulation control method to solve the attitude tracking and disturbance rejection problem of spacecraft for a class of persistent disturbances with unbounded energy which include the sinusoidal disturbance as a special case. The approach involves the integration of techniques from robust control, adaptive control, and robust output regulation theory.
\end{abstract}

\section{INTRODUCTION}

Attitude control of spacecraft systems has been a benchmark control problem and has been extensively studied under various assumptions and scenarios [1], [3], [7], [9], [10], [12], [13]. An important control method for disturbance attenuation of spacecraft systems is the $\mathcal{H}_{\infty}$ control method. Most $\mathcal{H}_{\infty}$ based methods can only attenuate the effect of disturbance on the output, quantified by $\mathcal{L}_{2}$ gain, to some degree. An exception was given recently in [9] where the authors employed the so-called inverse optimal adaptive control method to achieve global attitude tracking. A distinct feature of their approach as opposed to others is that their control law allows the $\mathcal{L}_{2}$ gain of the closed-loop system to be chosen arbitrarily small so as to achieve any level of $\mathcal{L}_{2}$ disturbance attenuation. Moreover, for the special class of disturbances with bounded energy, the control law given in [9] can further lead to asymptotic disturbance rejection, that is, the steady-state attitude tracking can be exactly achieved in the presence of disturbances. It should be noted that the inverse optimal adaptive control method was first developed in [8] without considering the external disturbances and plant uncertainties.

Nevertheless, in more practical situations, the external disturbances are not supposed to be energy bounded. For example, the sinusoidal function is not energy bounded. Therefore, an interesting problem is to design an attitude control law that can achieve global attitude tracking in the presence of mass parameter uncertainties and external disturbances with unbounded energy. The purpose of this paper is to develop a methodology for synthesizing a robust adaptive control law that achieves global attitude tracking for a spacecraft subject to unknown mass parameters and a class

Z. Chen is with the School of Electrical Engineering and Computer Science, The University of Newcastle, NSW 2308, Australia zhiyong. chen@newcastle.edu.au

J. Huang is with the Department of Mechanical and Automation Engineering, The Chinese University of Hong Kong, Shatin, N.T., Hong Kong jhuang@mae. cuhk . edu.hk

The first author was partially supported by the Early Career Researcher Grant G0187304. The second author was substantially supported by a grant from the Research Grants Council of the Hong Kong Special Administration Region (Project no.: CUHK4168/03E) and by the National Natural Science Foundation of China under grant No. 60374038 of disturbances which is a finite sum of sinusoidal functions with unknown amplitudes and phases.

Since the $\mathcal{H}_{\infty}$ control method can only achieve disturbance attenuation for disturbances with unbounded energy, the method in [7], [9], [10], [12] cannot be extended to achieve disturbance rejection. Therefore, we need to develop a new methodology to deal with the problem. First, the attitude control and disturbance rejection problem of the spacecraft will be formulated in Section II. The complete motion equation will be called System A(equations (1) - (4)). In order to convert the problem to a more tractable problem, we need to perform two major transformations. First, we make use of the results in [9], [14] to simplify our problem into a regulation problem of a class of nonlinear systems subject to linearly parameterized uncertain parameter and external disturbances. This class of systems will be presented in Section III and be called System B (equation (9)). Second, to identify the unknown disturbance in System $A$, in Section IV, we will make use of the robust output regulation theory as developed in [2], [4], [5] to reproduce the unknown external disturbance by a class of dynamic compensators known as internal models. Again by making use of the robust output regulation theory, we can introduce a set of coordinate and input transformation to obtain a so-called augmented system which will be called System C (equation (27) or (32)). Then we further show that the adaptive regulation problem of System C is solvable and its solution leads to that of the attitude control and disturbance rejection problem of the spacecraft system (System A). Finally, the paper is closed with some conclusions in Section VI.

\section{Plant Model and Problem Formulation}

In this section, we will first present the mathematical model of a rigid spacecraft, which can be found in many books, e.g., [3] and [11]. We will use the unit quaternion to represent the attitude of the spacecraft which is free of singularity. The quaternion is a vector defined by $q:=$ $\left[q_{1}, q_{2}, q_{3}, q_{4}\right]^{\top}$ where $q_{v}:=\left[q_{1}, q_{2}, q_{3}\right]^{\top}$ is called the vector part of $q$ and $q_{4}$ is the scalar part of $q$. The quaternion $q$ has the constraint $q_{v}^{\top} q_{v}+q_{4}^{2}=1$. In terms of the unit quaternion, the attitude kinematics is given by

$$
\begin{aligned}
\dot{q}_{v} & =\frac{1}{2}\left(q_{4} I_{3}+q_{v}^{\times}\right) \Omega \\
\dot{q}_{4} & =\frac{1}{2} q_{v}^{T} \Omega
\end{aligned}
$$

where $\Omega \in \mathbb{R}^{3}$ is the angular velocity of the body frame relative to a reference frame, ${ }^{\times}$is an operator on the three $\mathrm{di}$ - 
mensional vector $q_{v}$ such that $q_{v}^{\times}:=\left[\begin{array}{ccc}0 & -q_{3} & q_{2} \\ q_{3} & 0 & -q_{1} \\ -q_{2} & q_{1} & 0\end{array}\right]$. The dynamic equation of attitude motion is as follows:

$$
J \dot{\Omega}=-\Omega^{\times} J \Omega+u+d
$$

where $J:=\left[\begin{array}{lll}J_{11} & J_{12} & J_{13} \\ J_{12} & J_{22} & J_{23} \\ J_{13} & J_{23} & J_{33}\end{array}\right] \in \mathbb{R}^{3 \times 3}$ is constant, positive definite, overall inertia matrix of the integrated satellite system, $u \in \mathbb{R}^{3}$ is the control torque, and $d \in \mathbb{R}^{3}$ is the external disturbance. As in [9], we suppose the desired attitude motion is

$$
\begin{aligned}
\dot{q}_{d v} & =\frac{1}{2}\left(q_{d 4} I_{3}+q_{d v}^{\times}\right) \Omega_{d} \\
\dot{q}_{d 4} & =-\frac{1}{2} q_{d v}^{\top} \Omega_{d}
\end{aligned}
$$

where $\left[q_{d v}, q_{d 4}\right]^{\top}$ with $q_{d v}:=\left[q_{d 1}, q_{d 2}, q_{d 3}\right]^{\top}$ is the unit quaternion representing the target attitude and $\Omega_{d} \in \mathbb{R}^{3}$ is the target angular velocity.

The external disturbance considered in this paper is a combination of a constant and some sinusoidal functions, i.e., each component $d_{i}(t), i=1,2,3$, of the disturbance $d(t)$, can be represented as follows:

$$
d_{i}(t)=C_{i 0}+\Sigma_{j=1}^{n_{i}} C_{i j} \sin \left(\omega_{i j}+\Upsilon_{i j}\right)
$$

where $C_{i j}$ and $\Upsilon_{i j}$ are arbitrarily unknown amplitudes and phases, and $\omega_{i j}$ are known frequencies.

Now it is ready to describe the control objective of the paper as follows.

Attitude Tracking and Disturbance Rejection Problem (ATDRP): Given desired target angular velocity $\Omega_{d}$ : $[0, \infty) \rightarrow \mathbb{R}^{3}$ such that $\Omega_{d}$ and $\dot{\Omega}_{d}$ are bounded over $[0, \infty)$ and the desired attitude motion described by (3), design a state feedback control law, independent of the class of disturbances $d(t)$ generated by (4), such that, the state of the closed-loop system composed of (1), (2) and the control law is bounded, and

$$
\begin{aligned}
& \lim _{t \rightarrow \infty} q_{\text {error }}(t):=\lim _{t \rightarrow \infty}\left[\begin{array}{l}
q_{v}(t)-q_{d v}(t) \\
q_{4}(t)-q_{d 4}(t)
\end{array}\right]=0, \\
& \lim _{t \rightarrow \infty}\left(\Omega(t)-C \Omega_{d}(t)\right)=0 .
\end{aligned}
$$

Remark 2.1: The attitude tracking and disturbance attenuation problem has been studied in [9] in the sense that a controller is designed to achieve the following objective

$$
\lim _{t \rightarrow \infty} \sup _{t \leq \tau}\left\|q_{\text {error }}(\tau)\right\| \leq \gamma \lim _{t \rightarrow \infty} \sup _{t \leq \tau}\|d(\tau)\|
$$

with $\gamma>0$ a prescribed number. However, if $d(t)$ does not decay to zero as $t \rightarrow \infty$, then, the objective of the asymptotic disturbance rejection (5) cannot be achieved. In this paper, we aim at achieving attitude tracking and disturbance rejection for the class of disturbances described by (4).

\section{Problem Conversion}

Having defined the problem, we will further show that, by a series of transformations, the main problem can be converted into a well formulated global regulation problem. For this purpose, let us first define $s_{d}:=\operatorname{col}\left(q_{d v}, q_{d 4}, \Omega_{d}, \dot{\Omega}_{d}\right)$. Using the following standard coordinate transformation as introduced in [11],

$$
\begin{aligned}
e_{v} & =q_{d 4} q_{v}-q_{d v}^{\times} q_{v}-q_{4} q_{d v} \\
e_{4} & =q_{d v}^{\top} q_{v}+q_{4} q_{d 4} \\
\omega & =\Omega-C \Omega_{d}
\end{aligned}
$$

with $C=\left(1-2 e_{v}^{\top} e_{v}\right) I_{3}+2 e_{v} e_{v}^{\top}-2 e_{4} e_{v}^{\times}$, we have

$$
\begin{aligned}
\dot{e}_{v}= & \frac{1}{2}\left(e_{4} I_{3}+e_{v}^{\times}\right) \omega \\
\dot{e}_{4}= & -\frac{1}{2} e_{v}^{T} \omega \\
J \dot{\omega}= & -\left(\omega+C \Omega_{d}\right)^{\times} J\left(\omega+C \Omega_{d}\right)+J\left(\omega^{\times} C \Omega_{d}-C \dot{\Omega}_{d}\right) \\
& +u+d .
\end{aligned}
$$

It can be verified that the error $e:=\operatorname{col}\left(e_{v}, e_{4}\right)$ is constrained by $e_{v}^{\top} e_{v}+e_{4}^{2}=1$.

Remark 3.1: It has been proved in [14] that the objective (5) can be achieved if there exists a control law for system (7) such that $\lim _{t \rightarrow \infty} e_{v}(t)=0$ and $\lim _{t \rightarrow \infty} \omega(t)=0$. This fact can be used to further simplify our problem. In fact, as suggested in [9], the following coordinate transformation

$$
\omega=z-K e_{v}
$$

gives

$$
\begin{aligned}
\dot{e}_{v} & =\frac{1}{2}\left(e_{4} I_{3}+e_{v}^{\times}\right)\left(z-K e_{v}\right) \\
\dot{e}_{4} & =-\frac{1}{2} e_{v}^{T}\left(z-K e_{v}\right) \\
J \dot{z} & =-\left(\omega+C \Omega_{d}\right)^{\times} J\left(\omega+C \Omega_{d}\right)+J\left(\omega^{\times} C \Omega_{d}-C \dot{\Omega}_{d}\right) \\
& +J K\left[\frac{1}{2}\left(e_{4} I_{3}+e_{v}^{\times}\right) \omega\right]+u+d .
\end{aligned}
$$

We now show that the kinematics subsystem, i.e., (9a) and (9b) has some desirable stability property as follows.

Lemma 3.1: Consider the kinematics subsystem, i.e., (9a) and (9b), where $K$ is some symmetric positive definite matrix. Then for any piecewise continuous time function $z(t)$ defined for $t \geq 0$ satisfying $\lim _{t \rightarrow \infty} z(t)=0$, and any initial state $e(0)$, the solution of the subsystem is bounded for all $t \geq 0$ and $\lim _{t \rightarrow \infty} e_{v}(t)=0$.

Proof: First note that since $e_{v}^{\top} e_{v}+e_{4}^{2}=1$. $e_{v}$ and $e_{4}$ are always bounded. Define a set $\mathbb{B}(\epsilon)=\left\{x \in \mathbb{R}^{3} \mid\|x\| \leq \epsilon\right\}$. We need to prove that for any $\epsilon>0$, there exists a finite time $T \geq 0$ such that

$$
e_{v}(t) \in \mathbb{B}(\epsilon), \forall t \geq T \text {. }
$$

Let $k_{\min }:=\lambda_{\min }(K)$ be the smallest eigenvalue of $K$. Since $\lim _{t \rightarrow \infty} z(t)=0$, there exists a finite time $T_{1} \geq 0$ such that

$$
\|z(t)\| \leq \frac{1}{2} \epsilon k_{\min }, \forall t \geq T_{1} .
$$


If there exist no finite time $T_{2} \geq T_{1}$ such that $e_{v}\left(T_{2}\right) \notin$ $\mathbb{B}(\epsilon)$, then (10) holds with $T=T_{1}$ and the proof is completed. Thus, in what follows, we assume there exists a finite time $T_{2} \geq T_{1}$ such that $e_{v}\left(T_{2}\right) \notin \mathbb{B}(\epsilon)$. We will show this assumption leads to the following two claims:

(i) There exists a finite time $T>T_{2}$ such that $e_{v}(T) \in$ $\mathbb{B}(\epsilon)$ and $e_{v}(t) \notin \mathbb{B}(\epsilon), \forall T_{2} \leq t<T$ (that is, $T$ is the first time at which $e_{v}(t)$ enters $\mathbb{B}(\epsilon)$ );

(ii) $e_{v}(t) \in \mathbb{B}(\epsilon), \forall t \geq T$.

Satisfaction of (10) clearly follows from these two claims. Thus, we only need to prove these two claims.

Proof of Claim $i$ :

We first prove that

$$
\left\|e_{v}(t)\right\| \geq \epsilon \Longrightarrow \dot{e}_{4}(t) \geq \frac{1}{4} k_{\min } \epsilon^{2}>0, \forall t \geq T_{1} .
$$

Indeed, we have

$$
\frac{1}{2} e_{v}^{\top} K e_{v} \geq \frac{1}{2} k_{\min }\left\|e_{v}\right\|^{2} \geq \frac{1}{2} k_{\min }\left\|e_{v}\right\| \epsilon \geq\left\|e_{v}\right\|\|z\| \geq e_{v}^{\top} z .
$$

Using (9b), we have, $\forall t \geq T_{1}$,

$$
\begin{aligned}
\dot{e}_{4} & =\frac{1}{2} e_{v}^{\top} K e_{v}-\frac{1}{2} e_{v}^{\top} z \\
& \geq \frac{1}{2} k_{\min }\left\|e_{v}\right\| \epsilon-\frac{1}{4} k_{\min }\left\|e_{v}\right\| \epsilon \\
& =\frac{1}{4} k_{\min }\left\|e_{v}\right\| \epsilon \geq \frac{1}{4} k_{\min } \epsilon^{2}>0 .
\end{aligned}
$$

If (i) doesn't hold, then for all $t \geq T_{2}, e_{v}(t) \notin \mathbb{B}(\epsilon)$, that is, $\left\|e_{v}(t)\right\|>\epsilon$. From (11), $\dot{e}_{4}(t)$ is positive for all $t \geq T_{2}$, so there is a finite time $T_{3} \geq T_{2}$ such that

$$
e_{4}\left(T_{3}\right)>\sqrt{1-\epsilon^{2}} \text {. }
$$

In fact, it suffices to choose $T_{3} \geq 4 \frac{\sqrt{1-\epsilon^{2}}-e_{4}\left(T_{2}\right)}{\epsilon^{2} k_{\min }}+T_{2}$. As a result,

$$
\left\|e_{v}\left(T_{3}\right)\right\|=\sqrt{1-e_{4}^{2}\left(T_{3}\right)}<\epsilon,
$$

i.e., $e_{v}\left(T_{3}\right) \in \mathbb{B}(\epsilon)$. Now, the proof of claim (i) is completed.

\section{Proof of Claim ii:}

We first prove $e_{4}(T)>0$ as follows:

On one hand, in (i) we know that for $T_{2} \leq t<T, e_{v}(t) \notin$ $\mathbb{B}(\epsilon)$, hence $\dot{e}_{4}(t)>0$. It implies that $e_{4}(t)$ is increasing during $T_{2} \leq t<T$. Thus $e_{4}(T)>e_{4}\left(T_{2}\right)$.

On the other hand, $e_{v}(T) \in \mathbb{B}(\epsilon)$ gives $\left\|e_{v}(T)\right\| \leq \epsilon$, and $e_{v}\left(T_{2}\right) \notin \mathbb{B}(\epsilon)$ gives $\left\|e_{v}\left(T_{2}\right)\right\|>\epsilon$. As a result, $\left\|e_{v}(T)\right\|<$ $\left\|e_{v}\left(T_{2}\right)\right\|$, hence $\left|e_{4}(T)\right|>\left|e_{4}\left(T_{2}\right)\right|$.

From $e_{4}(T)>e_{4}\left(T_{2}\right)$ and $\left|e_{4}(T)\right|>\left|e_{4}\left(T_{2}\right)\right|$, we have $e_{4}(T)>0$.

Suppose $T_{4} \geq T$ be the first time the trajectory of $e_{v}$ is at the edge of $\mathbb{B}(\epsilon)$, that is, $e_{v}(t) \in \mathbb{B}(\epsilon), \forall T \leq t \leq T_{4}$. As a result, during $T \leq t \leq T_{4}$, we have $\left\|e_{v}(t)\right\| \leq \epsilon$, and $\left|e_{4}(t)\right| \geq \sqrt{1-\epsilon^{2}}$.

From $e_{4}(T)>0$, the continuousness of $e_{4}(t)$ and $\left|e_{4}(t)\right| \geq \sqrt{1-\epsilon^{2}}$, we have $e_{4}(t)>0, \forall T \leq t \leq T_{4}$.

At the edge of $\mathbb{B}(\epsilon)$, we have $\left\|e_{v}\left(T_{4}\right)\right\|=\epsilon$. By (11), we have $\dot{e}_{4}\left(T_{4}\right)>0$.

Thus, we have $e_{4}\left(T_{4}\right)>0$ and $\dot{e}_{4}\left(T_{4}\right)>0$, which imply that, at the edge of $\mathbb{B}(\epsilon)$, the time derivative of $\left|e_{4}(t)\right|$ is positive, hence, the time derivative of $\left\|e_{v}(t)\right\|$ is negative. As a result, the trajectories $e_{v}(t)$ cannot cross the edge of $\mathbb{B}(\epsilon)$ from inside to outside. Claim (ii) is thus proved.

By this lemma and Remark 3.1, we immediately obtain the following result.

Theorem 3.1: Suppose there exists a dynamic state feedback control law of the form

$$
\begin{aligned}
u & =k_{u}\left(e, z, s_{d}, \Theta\right) \\
\dot{\Theta} & =\varphi\left(e, z, s_{d}, \Theta\right),
\end{aligned}
$$

where both $k_{u}(\cdot, \cdot, \cdot, \cdot)$ and $\varphi(\cdot, \cdot, \cdot, \cdot)$ are globally defined smooth functions, such that, for any initial condition, the solution of the closed-loop system composed of (9) and (12) is bounded and

$$
\lim _{t \rightarrow \infty} z(t)=0
$$

Then, for any initial conditions, the states of the closed-loop system composed of (7) and (12) are bounded and

$$
\lim _{t \rightarrow \infty} e_{v}(t)=0 \text { and } \lim _{t \rightarrow \infty} \omega(t)=0 .
$$

Hence, the attitude tracking and disturbance rejection problem is solved by the controller (12) under the coordinate transformation (6) and (8).

By this theorem, we have simplified the ATDRP described in Section II to the global stabilization problem of the dynamic subsystem (9).

\section{PREliminary Manipulation on Dynamics SUBSYSTEM}

System (9) contains two types of uncertainties, namely, the parameter uncertainty of the elements of the matrix $J$, and the unknown disturbance $d(t)$. Without $d(t)$, the stabilization of (9) can be achieved by the standard adaptive control approach. On the other hand, without the uncertainty $J$, the stabilization of (9) can be achieved by the internal model based approach. However, with the presence of both types of uncertainties, neither the adaptive control approach nor the internal model based approach works alone. To overcome this difficulty, we will tackle our problem in two steps. In this section, we introduce a dynamic compensator that can asymptotically estimate the unknown disturbance in some sense. This dynamic compensator is motivated from the robust output regulation theory. The dynamic compensator together with system (9) is called the augmented system which is in the form of (27). In next section, we will develop an adaptive controller to stabilize the augmented system (27), thus leading to the solution of our problem.

Let us first put system (9) in a more standard form. As suggested in [9], for any vector $a:=\left[a_{1}, a_{2}, a_{3}\right]^{\top} \in \mathbb{R}^{3}$, we have $J a=L(a)\left[\begin{array}{llllll}J_{11} & J_{22} & J_{33} & J_{23} & J_{13} & J_{12}\end{array}\right]^{\top}$ where $L(a):=\left[\begin{array}{cccccc}a_{1} & 0 & 0 & 0 & a_{3} & a_{2} \\ 0 & a_{2} & 0 & a_{3} & 0 & a_{1} \\ 0 & 0 & a_{3} & a_{2} & a_{1} & 0\end{array}\right]$. When some entries of $J$ are unknown, there exists an unknown vector $\delta$ with dimension $0 \leq n_{\delta} \leq 6$ such that

$$
\left[\begin{array}{llllll}
J_{11} & J_{22} & J_{33} & J_{23} & J_{13} & J_{12}
\end{array}\right]^{\top}=\bar{L}_{1} \delta+\bar{L}_{0}
$$


for some known matrices $\bar{L}_{1} \in \mathbb{R}^{6 \times n_{\delta}}, \bar{L}_{0} \in \mathbb{R}^{6 \times 1}$. Let $L_{1}(a)=L(a) \bar{L}_{1}$ and $L_{0}(a)=L(a) \bar{L}_{0}$, we have $J a=$ $L_{1}(a) \delta+L_{0}(a)$. Therefore, we have

$$
\begin{aligned}
& -\left(\omega+C \Omega_{d}\right)^{\times} J\left(\omega+C \Omega_{d}\right)+J\left(\omega^{\times} C \Omega_{d}-C \dot{\Omega}_{d}\right) \\
= & F_{1}\left(e, z, s_{d}\right) \delta+F_{0}\left(e, z, s_{d}\right),
\end{aligned}
$$

and

$$
J K\left(\frac{1}{2}\left(e_{4} I_{3}+e_{v}^{\times}\right) \omega\right)=G_{1}(e, z) \delta+G_{0}(e, z),
$$

where

$$
\begin{aligned}
F_{1}\left(e, z, s_{d}\right):=\quad & -\left(\omega+C \Omega_{d}\right)^{\times} L_{1}\left(\omega+C \Omega_{d}\right) \\
& +L_{1}\left(\omega^{\times} C \Omega_{d}-C \dot{\Omega}_{d}\right) \\
F_{0}\left(e, z, s_{d}\right):=\quad & -\left(\omega+C \Omega_{d}\right)^{\times} L_{0}\left(\omega+C \Omega_{d}\right) \\
& +L_{0}\left(\omega^{\times} C \Omega_{d}-C \dot{\Omega}_{d}\right) \\
G_{1}(e, z):= & \frac{1}{2} L_{1}\left(K\left(e_{4} I_{3}+e_{v}^{\times}\right) \omega\right) \\
G_{0}(e, z):= & \frac{1}{2} L_{0}\left(K\left(e_{4} I_{3}+e_{v}^{\times}\right) \omega\right) .
\end{aligned}
$$

It is noted that in general $F_{1}\left(e, 0, s_{d}\right) \neq 0, F_{0}\left(e, 0, s_{d}\right) \neq 0$, $G_{1}(e, 0) \neq 0$, and $G_{0}(e, 0) \neq 0$.

As a result, the subsystem $(9 \mathrm{c})$ becomes

$$
\begin{aligned}
J \dot{z}= & \left(F_{1}\left(e, z, s_{d}\right)+G_{1}(e, z)\right) \delta+F_{0}\left(e, z, s_{d}\right) \\
& +G_{0}(e, z)+u+d .
\end{aligned}
$$

To simplify (16), applying the input transformation $u=\bar{u}-$ $F_{0}\left(e, z, s_{d}\right)-G_{0}(e, z)$ to (16) gives

$$
J \dot{z}=\left(F_{1}\left(e, z, s_{d}\right)+G_{1}(e, z)\right) \delta+\bar{u}+d .
$$

To handle the uncertain disturbance $d(t)$, note that, for each $i=1,2,3$, there exist positive integers $r_{i}$ and real numbers $a_{i, 1}, \cdots, a_{i, r_{i}}$ such that

$$
\begin{array}{r}
\frac{d^{r_{i}} d_{i}(t)}{d t^{r_{i}}}-a_{i, 1} d_{i}(t)-a_{i, 2} \frac{d d_{i}(t)}{d t}-\cdots \\
-a_{i, r_{i}} \frac{d^{\left(r_{i}-1\right)} d_{i}(t)}{d t^{\left(r_{i}-1\right)}}=0 .
\end{array}
$$

Let $T_{i}$ be any nonsingular matrix of dimension $r_{i}$, and

$$
\theta_{i}(t)=T_{i}\left[\begin{array}{llll}
d_{i}(t) & \dot{d}_{i}(t) & \ldots & \frac{d^{\left(r_{i}-1\right)} d_{i}(t)}{d t^{\left(r_{i}-1\right)}}
\end{array}\right]^{\top}
$$

Then, we have

$$
\dot{\theta}_{i}(t)=T_{i} \Phi_{i} T_{i}^{-1} \theta_{i}(t), d_{i}(t)=\Psi_{i} T_{i}^{-1} \theta_{i}
$$

where

$$
\begin{aligned}
\Phi_{i} & =\left[\begin{array}{ccccc}
0 & 1 & 0 & \cdots & 0 \\
0 & 0 & 1 & \cdots & 0 \\
\vdots & \vdots & \vdots & \cdots & \vdots \\
0 & 0 & 0 & \cdots & 1 \\
a_{i, 1} & a_{i, 2} & a_{i, 3} & \cdots & a_{i, r_{i}}
\end{array}\right] \\
\Psi_{i} & =\left[\begin{array}{llll}
1 & 0 & \cdots & 0
\end{array}\right] .
\end{aligned}
$$

Using (20), it is possible to overcome the uncertainty caused by $d(t)$ by introducing a dynamic compensator as follows.
Let $M_{i} \in \mathbb{R}^{r_{i} \times r_{i}}$ and $N_{i} \in \mathbb{R}^{r_{i} \times 1}$ be a pair of controllable matrices with $M_{i}$ Hurwitz. Then, there exists a nonsingular matrix $T_{i}$ satisfying the Sylvester equation

$$
T_{i} \Phi_{i}-M_{i} T_{i}=N_{i} \Psi_{i}
$$

since the pair $\left(\Phi_{i}, \Psi_{i}\right)$ is observable. Let

$$
\begin{aligned}
\theta & :=\operatorname{col}\left(\theta_{1}, \theta_{2}, \theta_{3}\right) \\
M & :=\operatorname{block} \operatorname{diag}\left(M_{1}, M_{2}, M_{3}\right) \\
N & :=\operatorname{block} \operatorname{diag}\left(N_{1}, N_{2}, N_{3}\right) .
\end{aligned}
$$

Then we can define a dynamic compensator as follows:

$$
\dot{\eta}=M \eta+N \bar{u}-P_{0}(z)
$$

where $P_{0}(z)$ with $P_{0}(0)=0$ is some sufficiently smooth function to be determined later.

Consider the augmented system composed of (17) and (23). Under the following coordinate transformation,

$$
\bar{\eta}=\eta-\theta, \tilde{u}=\bar{u}-\Psi T^{-1} \eta
$$

we have

$$
\begin{aligned}
\dot{\bar{\eta}} & =\left(M+N \Psi T^{-1}\right) \bar{\eta}+N \tilde{u}-P_{0}(z) \\
J \dot{z} & =\left\{F_{1}\left(e, z, s_{d}\right)+G_{1}(e, z)\right\} \delta+\Psi T^{-1} \bar{\eta}+\tilde{u} .
\end{aligned}
$$

Remark 4.1: The compensator (23) is motivated from the robust output regulation theory as can be found in [5]. However, when $\delta \neq 0$, the disturbance rejection of system (17) cannot be viewed as a robust output regulation problem for two reasons. First, $s_{d}$ is not generated by some neutrally stable linear exosystem, and second, $F_{1}\left(e, 0, s_{d}\right) \neq$ $0, F_{0}\left(e, 0, s_{d}\right) \neq 0$. Thus (23) cannot be considered as an internal model of system (17). As a result, the framework for handling the robust output regulation problem established in [5] cannot be directly used on system (17). Nevertheless, the idea of the internal model design has still been utilized to handle the unknown disturbance $d(t)$.

In order to solve the attitude tracking and disturbance rejection problem, it suffices to find a control law to regulate the augmented system (25). For this purpose, let

$$
P_{0}(z)=M N L_{0}(z) .
$$

Then a further transformation $\tilde{\eta}=\bar{\eta}-N J z$ gives

$$
\begin{aligned}
\dot{\tilde{\eta}}= & M \tilde{\eta}+P_{1}\left(e, z, s_{d}\right) \delta \\
J \dot{z}= & \left(F_{1}\left(e, z, s_{d}\right)+G_{1}(e, z)\right) \delta+\Psi T^{-1} \tilde{\eta}+\Psi T^{-1} N J z \\
& +\tilde{u},
\end{aligned}
$$

with

$P_{1}\left(e, z, s_{d}\right):=M N L_{1}(z)-N\left[F_{1}\left(e, z, s_{d}\right)+G_{1}(e, z)\right]$.

We have now completed the conversion of the attitude tracking and disturbance rejection problem for the spacecraft into the regulation problem for system (27). This result is summarized as follows.

Lemma 4.1: If there exists a control law

$$
\begin{aligned}
\tilde{u} & =k_{\tilde{u}}\left(e, z, s_{d}, \eta, \Theta_{2}\right) \\
\dot{\Theta}_{2} & =\varphi_{2}\left(e, z, s_{d}, \eta, \Theta_{2}\right)
\end{aligned}
$$


such that the state of the system composed of (27) and (29 is bounded and $\lim _{t \rightarrow \infty} z(t)=0$. Then the attitude tracking and disturbance rejection problem is solved by a control law of the form (12) where $\Theta:=\operatorname{col}\left(\Theta_{1}, \Theta_{2}\right), \Theta_{1}=\eta$ and

$$
k_{u}\left(e, z, s_{d}, \Theta\right)=k_{\tilde{u}}\left(e, z, s_{d}, \eta, \Theta_{2}\right)+F_{0}\left(e, z, s_{d}\right)+G_{0}(e, z) \text {. }
$$

\section{Main Result}

So far we have converted the attitude tracking and disturbance rejection problem to a problem of designing a control law (29) such that the state of the system composed of (27) and (29) is bounded and $\lim _{t \rightarrow \infty} z(t)=0$. This can be considered as a regulation problem. Since $\delta$ is uncertain, we will use an adaptive scheme to deal with it. First we will perform a linear parameterization $J z=L_{1}(z) \delta+L_{0}(z)$, and utilize a feedforward control

$$
\tilde{u}=\hat{u}-\Psi T^{-1} N L_{0}(z)
$$

Then, system (27) becomes

$$
\begin{aligned}
\dot{\tilde{\eta}}= & M \tilde{\eta}+P_{1}\left(e, z, s_{d}\right) \delta \\
J \dot{z}= & {\left[F_{1}\left(e, z, s_{d}\right)+G_{1}(e, z)+\Psi T^{-1} N L_{1}(z)\right] \delta } \\
& +\Psi T^{-1} \tilde{\eta}+\hat{u} .
\end{aligned}
$$

Using a more compact notation $x_{1}=\tilde{\eta}, x_{2}=z, u=\hat{u}$, $\mu=\delta, w(t)=\operatorname{col}\left(e(t), s_{d}(t)\right)$ and

$$
\begin{aligned}
y_{m} & =\operatorname{col}\left(x_{2}, e, s_{d}\right) \\
A & =\Psi T^{-1} \\
f\left(y_{m}\right) & =P_{1}\left(e, z, s_{d}\right) \\
g\left(y_{m}\right) & =F_{1}\left(e, z, s_{d}\right)+G_{1}(e, z)+\Psi T^{-1} N L_{1}(z),
\end{aligned}
$$

we can put system (31) in the following form:

$$
\begin{aligned}
\dot{x}_{1} & =M x_{1}+f\left(y_{m}\right) \mu \\
J \dot{x}_{2} & =A x_{1}+g\left(y_{m}\right) \mu+u \\
y & =x_{2}
\end{aligned}
$$

with $x_{1} \in \mathbb{R}^{n_{1}}, x_{2} \in \mathbb{R}^{n_{2}}$ and $\mu \in \mathbb{R}^{n_{\mu}}$.

Now all we need to do is to solve the global regulation problem of system (32) by measurement output $\left(y_{m}\right)$ feedback control, i.e., to find a control law which depends on $y_{m}$ only such that, for any initial condition of the closed-loop system, the state of the closed-loop system is bounded and $\lim _{t \rightarrow \infty} y(t)=0$.

The regulation problem of system (32) poses specific difficulty because of the presence of $f\left(y_{m}\right) \mu$ and $g\left(y_{m}\right) \mu$ which do not vanish even when $x_{1}=0, x_{2}=0, u=0$. Thus we need to develop a dynamic coordination transformation technique to circumvent this difficulty. In fact, we have the following result.

Proposition 5.1: Consider system (32) and assume that $e(t)$ and $s_{d}(t)$ are bounded piecewise continuous time functions for $t \geq 0$. Then, there exists a control law depending on $y_{m}$ only such that, for any initial condition of the closedloop system, the state of the closed-loop system is bounded and $\lim _{t \rightarrow \infty} y(t)=0$. system

Proof: Let $\zeta \in \mathbb{R}^{n_{1} \times n_{\mu}}$ be produced by an auxiliary

$$
\dot{\zeta}=M \zeta+f\left(y_{m}\right) .
$$

Let $\bar{x}_{1}=x_{1}-\zeta \mu$. Direct calculation shows that

$$
\begin{aligned}
\dot{\bar{x}}_{1} & =M x_{1}+f\left(y_{m}\right) \mu-\left[M \zeta+f\left(y_{m}\right)\right] \mu=M \bar{x}_{1} . \\
J \dot{x}_{2} & =A \bar{x}_{1}+\rho\left(y_{m}, \zeta\right) \mu+u
\end{aligned}
$$

where $\rho\left(y_{m}, \zeta\right):=A \zeta+g\left(y_{m}\right)$.

It is noted that in system (34), the uncertainty term $f\left(y_{m}\right) \mu$ disappears while the newly introduced variable $\zeta$ is produced by a known function $f$ and matrix $M$. Therefore, system (33) and (34) is in a standard form as studied, e.g., in [6], and the regulation of this system can be handled as follows. Design a control law

$$
u=-\rho\left(y_{m}, \zeta\right) \hat{\mu}-K x_{2}
$$

where the vector $\hat{\mu}$ is used to estimate $\mu$ and $K$ is any symmetric positive definite matrix $K$ (let $K_{\min }>0$ be the smallest eigenvalue). And design an update law

$$
\dot{\hat{\mu}}=\Lambda \rho^{\top}\left(y_{m}, \zeta\right) x_{2}
$$

where $\Lambda$ is any diagonal matrix with positive diagonal entries which is used to modify update rate.

Now, let $Q$ be the symmetric positive definite matrix satisfying $Q M+M^{\top} Q=-I$ and pick a real number $\epsilon \geq\|A\|^{2} / K_{\min }$. By choosing

$$
V\left(\bar{x}_{1}, x_{2}, \tilde{\mu}\right)=\epsilon \bar{x}_{1}^{\top} Q \bar{x}_{1}+\frac{1}{2} x_{2}^{\top} J x_{2}+\frac{1}{2} \tilde{\mu}^{\top} \Lambda^{-1} \tilde{\mu}
$$

with $\tilde{\mu}=\hat{\mu}-\mu$, we have, along (33) and (34),

$$
\begin{aligned}
& \frac{d V\left(\bar{x}_{1}, x_{2}, \tilde{\mu}\right)}{d t} \\
= & -\epsilon\left\|\bar{x}_{1}\right\|^{2}+x_{2}^{\top} A \bar{x}_{1}-x_{2}^{\top} K x_{2} \\
& +\tilde{\mu}^{\top}\left[-\rho^{\top}\left(y_{m}, \zeta\right) x_{2}+\Lambda^{-1} \tilde{\tilde{\mu}}\right] \\
\leq & -\epsilon\left\|\bar{x}_{1}\right\|^{2}+\frac{1}{2 \epsilon}\left\|x_{2}^{\top} A\right\|^{2}+\frac{1}{2} \epsilon\left\|\bar{x}_{1}\right\|^{2}-x_{2}^{\top} K x_{2} \\
\leq & -a\left(\bar{x}_{1}, x_{2}\right)
\end{aligned}
$$

with $a\left(\bar{x}_{1}, x_{2}\right):=\frac{1}{2} \epsilon\left\|\bar{x}_{1}\right\|^{2}+\frac{1}{2} x_{2}^{\top} K\left(x_{2}\right)$. Thus, the states $\bar{x}_{1}, x_{2}$ and $\tilde{\mu}$ are bounded, so is the output $y_{m}$. As a result, the state $\zeta$ is bounded, and hence $x_{1}$ is bounded. Moreover, the function $V\left(\bar{x}_{1}, x_{2}, \tilde{\mu}\right)$ is lower bounded, and $a\left(\bar{x}_{1}, x_{2}\right)$ is uniformly continuous in $t$. By Barbalat's lemma, $\lim _{t \rightarrow \infty} x_{2}(t)=0$. The proof is thus completed.

Now applying Proposition 5.1 to system (31) and noting $e$ and $s_{d}$ are always bounded shows that the following control law

$$
\begin{aligned}
\hat{u} & =-K_{z} z-\rho\left(y_{m}, \zeta\right) \hat{\delta} \\
\dot{\zeta} & =M \zeta+P_{1}\left(e, z, s_{d}\right) \\
\dot{\hat{\delta}} & =\Lambda \rho^{\top}\left(y_{m}, \zeta\right) z
\end{aligned}
$$

is such that the state of the closed-loop system composed of (31) and (37) is bounded and $\lim _{t \rightarrow \infty} z(t)=0$. Hence, by Theorem 3.1 and Lemma 4.1, the attitude tracking and 
disturbance rejection problem formulated in this paper is solved by a controller of the form (12), which consists of (29) and (23). And (29) is in the form of (30) and (37) with $\Theta_{2}=\operatorname{col}(\zeta, \hat{\delta})$. Thus, we have reached our main result as follows.

Theorem 5.1: The attitude tracking and disturbance rejection problem formulated in this paper is solvable.

Simulation is conducted for demonstrating the performance of the control law designed above. The model of the spacecraft is taken from [1] where the inertia matrix

$$
J=\left[\begin{array}{ccc}
J_{11} & 1.2 & 0.9 \\
1.2 & 17 & 1.4 \\
0.9 & 1.4 & 15
\end{array}\right]
$$

which contains an unknown parameter $\delta=J_{11}$. We can calculate $F_{0}\left(e, z, s_{d}\right), F_{1}\left(e, z, s_{d}\right), G_{0}(e, z)$, and $G_{1}(e, z)$ from (15).

We consider the disturbance

$$
d(t)=\left[\begin{array}{l}
d_{1}(t) \\
d_{2}(t) \\
d_{3}(t)
\end{array}\right]=\left[\begin{array}{l}
C_{1} \sin \left(\omega_{1}+\Upsilon_{1}\right) \\
C_{2} \sin \left(\omega_{2}+\Upsilon_{2}\right) \\
C_{2} \sin \left(\omega_{3}+\Upsilon_{3}\right)
\end{array}\right] .
$$

Suppose $\omega_{1}=0.1, \omega_{2}=0.2$ and $\omega_{3}=0.2$. It is easy to see that $\Phi_{i}=\left[\begin{array}{cc}0 & 1 \\ -\omega_{i}^{2} & 0\end{array}\right], \Psi_{i}=\left[\begin{array}{ll}1 & 0\end{array}\right]$. Letting

$$
M_{i}=\left[\begin{array}{cc}
0 & 1 \\
-3 & -2
\end{array}\right], N_{i}=\left[\begin{array}{l}
0 \\
1
\end{array}\right]
$$

gives

$$
T_{i}^{-1}=\left[\begin{array}{cc}
3-\omega_{i}^{2} & 2 \\
-2 \omega_{i}^{2} & 3-\omega_{i}^{2}
\end{array}\right] .
$$

Hence, $\Psi_{i} T_{i}^{-1}=\left[\begin{array}{cc}3-\omega_{i}^{2} & 2\end{array}\right]$. Now, we can obtain $P_{0}(z)$ and $P_{1}\left(e, z, s_{d}\right)$ from (26) and (28), respectively. In particular, we note that $P_{1}\left(e, z, s_{d}\right) \in \mathbb{R}^{6 \times 1}$. Then, we can take $\eta \in \mathbb{R}^{6 \times 1}$ and $\zeta \in \mathbb{R}^{6 \times 1}$. Now, it is ready to give the control law (29) given by (30) and (37), hence the overall control law (12) consists of (23) and (29). In particular, we choose the gains of the control law as $K=3 I_{3}, K_{z}=20 I_{3}$, and the update rate matrix $\Lambda=I_{3}$.

To demonstrate the effectiveness of the above control law, we suppose the target angular velocity $\Omega_{d}$ is

$$
\Omega_{d}(t)=\left[\begin{array}{l}
0.05 \sin (1 \pi t / 100) \\
0.05 \sin (2 \pi t / 100) \\
0.05 \sin (3 \pi t / 100)
\end{array}\right]
$$

and the initial target unit quaternion is $q_{d}(0)=[0,0,0,1]^{\top}$. The initial attitude orientation of the spacecraft is $q(0)=$ $[0.3,-0.2,-0.3,0.8832]^{\top}$, and the initial value of the angular velocity is $\Omega(0)=[0,0,0]$. The disturbance magnitudes are $C_{1}=1, C_{2}=2$ and $C_{3}=6$. The nominal value of parameter $J_{11}=40$. The initial state of the update law is $\hat{\delta}(0)=10$, and the initial values of the remaining states are chosen as 0 . The simulation results for state $q_{1}$ are shown in Fig 1 . Similar tracking performance for $q_{2}, q_{3}$ and $q_{4}$ are achieved as well.
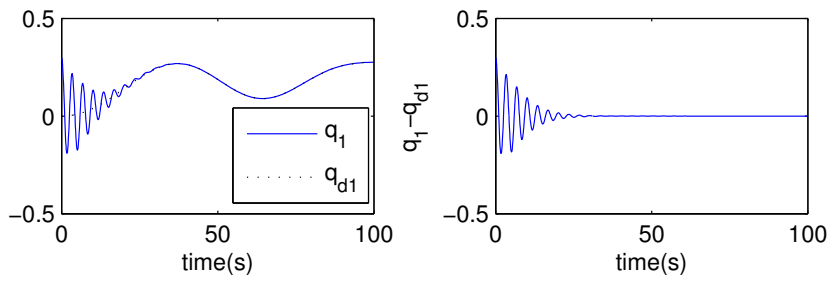

Fig. 1. Profile of states

\section{CONCLUSION}

In this paper, we have presented a result on the attitude tracking and disturbance rejection problem of the spacecraft system subject to plant uncertainty. A specific contribution of our approach is that our control law has achieved asymptotic rejection of a class of external disturbances with unbounded energy. Our approach has integrated techniques from robust control, adaptive control, and output regulation theory.

\section{REFERENCES}

[1] J. Ahmed, V. T. Coppola, and D. Bernstein, " Adaptive Asymptotic Tracking of Spacecraft Attitude Motion with Inertia Matrix Identification," Journal of Guidance, Control and Dynamics, vol. 21, pp. 684-691, 1998.

[2] C. I. Byrnes, F. Delli Priscoli, A. Isidori, and W. Kang, "Structurally stable output regulation of nonlinear systems," Automatica, vol. 33, pp. 369-385, 1997.

[3] W. E. Dixon, A. Behal, D. M. Dawson, and S. P. Nagarkatti, Nonlinear Control of Engineering Systems: A Lyapunov-Based Approach, Birkhäuser, 2003.

[4] J. Huang, "Asymptotic tracking and disturbance rejection in uncertain nonlinear systems," IEEE Transactions on Automatic Control, vol 40. pp. 1118-1122, 1995.

[5] J. Huang and Z. Chen, "A general framework for tackling the output regulation problem," IEEE Transactions on Automatic Control, No. 12, pp. 2203 - 2218, Dec. 2004.

[6] Z. P. Jiang and L. Praly, "Design of robust adaptive controllers for nonlinear systems with dynamic uncertainties," Automatica, vol.34, pp.825-840, 1998.

[7] W. Kang, "Nonlinear $H_{\infty}$ control and its applications to rigid spacecraft," IEEE Transactions on Automatic Control, vol. 40, no. 7, pp. 1281-1285, 1995

[8] M. Kristic and P. Tsiotras, "Inverse optimal stabilization of a rigid spacecraft," IEEE Transactions on Automatic Control, vol. 44. pp.1042-1049, 1999.

[9] W. Luo, Y.-C. Chu, and K.-V. Ling "Inverse Optimal adaptive control for attitude tracking of spacecraft," IEEE Transactions on Automatic Control, vol. 50, pp. 1639 - 1654, Nov. 2005.

[10] W. Luo, Y.-C. Chu, and K.-V. Ling "Optimal adaptive attitude tracking control of rigid spacecraft" Proceedings of the Mediterranean Conference on Control and Automation, Kusadasi, Turkey, June 2004.

[11] M. J. Sidi, Spacecraft Dynamics and Control, Cambridge University Press, 1997.

[12] C.-D. Yang and C.-C. Kung, "Nonlinear $H_{\infty}$ flight control of general six-degree-of-freedom motions," Journal of Guidance, Control and Dynamics, vol. 23, no. 2, pp. 278-288, 2000.

[13] H. Yoon and P. Tsiotras, "Spacecraft adaptive attitude and power tracking with variable speed control moment gyroscopes," Journal of Guidance, Control and Dynamics, vol. 25, no. 6, pp. 1081-1090, 2002.

[14] J. S.-C. Yuan, "Closed-loop manipulator control using quaternion feedback," IEEE Transactions on Automatic Control, vol. 4, pp. 434440, 1988. 